Mathematical Modelling and Analysis

Volume 22 Number 6, November 2017, 809-826

https://doi.org/10.3846/13926292.2017.1384765

(c) Vilnius Gediminas Technical University, 2017
Publisher: Taylor\&Francis and VGTU

http://www.tandfonline.com/TMMA

ISSN: $1392-6292$

eISSN: 1648-3510

\title{
Triply Resonant Double Diffusive Convection in a Fluid Layer
}

\author{
Akil J. Harfash ${ }^{a}$ and Fahad K. Nashmi ${ }^{a}$ \\ ${ }^{a}$ Department of Mathematics, College of Sciences, University of Basrah \\ Basrah, Iraq \\ E-mail(corresp.): akilharfash@gmail.com \\ E-mail: fahadkamel1987@yahoo.com
}

Received April 22, 2017; revised September 19, 2017; published online November 15, 2017

\begin{abstract}
We study the problem of double-diffusive convection in a horizontal plane fluid layer when there is a heat sink/source which is linear in the vertical coordinate which is in the opposite direction to gravity. The thresholds for linear instability are found and compared to those derived by a global nonlinear energy stability analysis. A region is discovered where a very sharp increase in Rayleigh number is observed. In addition to a linearized instability analysis, two global (unconditional) nonlinear stability thresholds are derived.
\end{abstract}

Keywords: double-diffusive, three layer, resonance, thermal insulation.

AMS Subject Classification: 76E06; 76M22.

\section{Nomenclature}

$\left(x_{1}, x_{2}, x_{3}\right)=(x, y, z)-$ Cartesian coordinates, $\mathbf{v}$ - velocity, $p$ - pressure,

$T$ - temperature, $C$ - concentration, $\mathbf{u}$ - dimensionless velocity,

$\mathcal{P}$ - dimensionless pressure, $\theta$ - dimensionless temperature,

$\phi$ - dimensionless concentration, $g$ - gravitational acceleration,

$\nu$ - dynamic viscosity, $\Delta$ - Laplacian, $\kappa_{t}$ - thermal diffusivity,

$\kappa_{c}$ - the solute diffusivity, $P_{r} \& P_{s}$ - Prandtl numbers,

$h(x, y)$ - plane-tiling planform, $\left(\bar{v}_{i}, \bar{p}, \bar{c}, \bar{T}\right)$ - steady state solution,

$\left(u_{i}, \pi, \phi, \theta\right)$ - perturbation, $\rho$ - density, $\rho_{0}$ - reference density,

$T_{m}$ - reference temperature, $C_{m}$ - reference concentration,

$\alpha_{t}$ - thermal expansion coefficient, $\alpha_{c}$ - solute expansion coefficient,

$R a=R_{t}^{2}$ - thermal Rayleigh number, $R_{c}$ - solute Rayleigh number,

$R a_{L}$ - critical Rayleigh number for linear instability theory,

$R a_{E}$ - critical Rayleigh number for the nonlinear stability theory,

$a$ - horizontal wavenumber, $a_{x}$ - wavenumbers in the $x$ direction,

$a_{y}$ - wavenumbers in the $y$ direction, $Q$ - internal heat source, $\sigma$ - growth rate. 


\section{Introduction}

Combined heat and mass transfer problems are significant in a range of processes, for example: drying, evaporation from the surface of a body of water, energy transfer in a wet cooling tower and the flow in a desert cooler, heat and mass transfers occur simultaneously. Possible applications of this type of flow can be found in such industries as the power industry, where among electric power generation methods is one in which electrical energy is extracted directly from a moving conducting fluid, cf., Straughan [19] and the references therein.

Some very informative analyses of resonance in thermal convection have been published recently. That is to say, instability in one part of a fluid layer may arise simultaneously with instability in another part of the layer. This resonance can lead to unusually high Rayleigh numbers at the onset of thermal convection and has considerable bearing upon the heat transfer industry. Typically, a high Rayleigh number may be accompany delaying or prohibiting heat transfer and plays an important role in insulation as a consequence. However, low Rayleigh numbers may be necessary when rapid heat transfer is called for, such as in cooling pipes used in many modern devices such as computers. A mathematical analysis of such resonance will be useful in the authors opinion, with regard to the potential implications of resonance in the energy sector, especially with nano-devices (for example, [3] and [16]).

The objective of this study is to consider double diffusive convection in a plane layer when the density-temperature relation in the buoyancy term is quadratic. However, we allow a heat sink/source that varies linearly vertical height $z$. This can give rise to the layer effectively splitting into three sublayers. In the lowest one, the fluid tends to be convectively unstable. In the intermediate layer the fluid will be gravitationally stable. In the top layer instability of the fluid is again possible. This results in a problem where convection may commence in either the lowest layer, the upmost layer, or possibly in both sublayers simultaneously. In this case resonance between the upmost and lowest layers is possible. In all cases there is the possibility of penetrative convection where convective movement in one layer induces motion in an adjacent sublayer. In certain cases the critical Rayleigh number for thermal convection may display a very rapid increase, much greater than normal. This behaviour may have an application in energy research, for example in thermal insulation.

Resonance in double-diffusive convection likewise has many applications since interactions between the fluid layers may greatly increase the critical Rayleigh number threshold for the onset of convection, which in turn is of interest to the energy industry. In particular, the application of this problem can be found in: building design and heat transfer, magmas and in other fluids of Geophysical and Geophysics, astrophysics, oceanography, lava flows may be responsible for enhancing the rise of volcanic plumes into the Earths stratosphere, convective phenomena in both multicoinponent fluids and crystallizing fluid systems, and salt finger and diffusive interfaces.

Assessing the onset and type of convection is crucial in understanding this system, which can be achieved by analysing both the linear instability and nonlinear stability thresholds of the governing model. Comparing these thresh- 
olds allows for the assessment of the suitability of linear theory to predict the physics of the onset of convection. In order to establish stability results we turn our attention to the highly adaptable energy method [19]. Nonlinear energy methods are particular useful as they delimit the parameter region of possible subcritical instability (the region between the linear instability and nonlinear stability thresholds). Hence, quantifying the discrepancy between these two thresholds makes it possible to provide an assessment of the suitability of linear theory to predict the de-stabilisation of the double diffusive convection. Recent contributions on the study of convective instabilities in porous media include $[4,5,6,7,8,9,10,14,15,20,21,22,23]$.

In the next section, we present the governing equations of motion and derive the associated perturbation equations. We introduce the linear and nonlinear analysis of our system in Section 3 and 4, respectively. Since the stability analyses involve eigenvalue problems with non-constant coefficients these problems must be solved numerically and a suitable numerical method is described in Section 4. In Section 5, the numerical results for the linear theory and a direct comparison with those of the global nonlinear theories are presented.

\section{Basic Equations}

We suppose the fluid is contained in the plane layer $\{z \in(0, d)\} \times \mathbb{R}^{2}$, and is incompressible, although a Boussinesq approximation is employed in the buoyancy term in the momentum equation. The $z$ direction is denoted by the vector $k$ with $i, j, k$ being the standard Cartesian basis. Gravity acts in the negative $\mathrm{z}$ direction and we assume that the density $\rho$ is constant, everywhere except the body force. Then, the Navier-Stokes equation for the fluid motion is

$$
\rho_{0}\left(v_{i, t}+v_{j} v_{i, j}\right)=-p_{, i}+\rho_{0} \nu \Delta v_{i}-\rho_{0} k_{i} g \rho(T, C),
$$

where $\rho, \mathbf{v}, p, T, C$ are the density, velocity field, pressure, temperature, concentration of solute. Additionally, $\nu$ is the dynamic viscosity, $g$ is gravity, and $\mathbf{k}=(0,0,1)$. Throughout, we use standard indicial notation and the Einstein summation convention so that e.g. $v_{i, t}=\partial v_{i} / \partial t$, and $p_{, i}=\partial p / \partial x_{i}$, $v_{j} v_{i, j} \equiv(\mathbf{v} . \nabla) \mathbf{v}$, and $\Delta$ is the Laplacian. The balance of mass equation is

$$
v_{i, i}=0 .
$$

The heat equation governing the temperature field is defined as

$$
T_{, t}+v_{i} T_{, i}=\kappa_{t} \Delta T+Q(z),
$$

where $Q(z)=\xi_{1} z+\xi_{2}$ and $\kappa_{t}$ is the thermal diffusivity. The equation governing the evaluation of the solute concentration is

$$
C_{, t}+v_{i} C,{ }_{i}=\kappa_{c} \Delta C,
$$

where $\kappa_{c}$ is the solute diffusivity. The density $\rho$ is of the form

$$
\rho(T, C)=\left(1-\alpha_{t}\left(T-T_{m}\right)^{2}+\alpha_{c}\left(C-C_{m}\right)\right),
$$


where $\rho_{0}, T_{m}$ and $C_{m}$ are a reference density, temperature and concentration, respectively, and $\alpha_{t}$ and $\alpha_{c}$ are the coefficients for thermal and solute expansion, respectively, $\xi_{1}, \xi_{2}$ are constants to be defined later.

The model now consists of the six partial differential equations (2.1)-(2.4). On the boundaries $z=0, d$ the following boundary conditions are assumed to hold,

$$
C=C_{U}, \quad z=d ; \quad C=C_{L}, \quad z=0,
$$

where $C_{U}, C_{L}$ are constants and $C_{L}>C_{U}$. The temperature is kept constant on the boundaries $z=0, d$, with $T=T_{L}$ at $z=0$, and $T=T_{m}$ at $z=d$, $T_{L}>T_{m}$. In this situation there is a possibility of convective instability driven by the sublayer $\left(0, \beta_{1} d\right)$ or by $\left(\beta_{2} d, d\right)$, where $z=\beta_{1} d$ and $z=\beta_{2} d$ are turning points for $T(z)$.

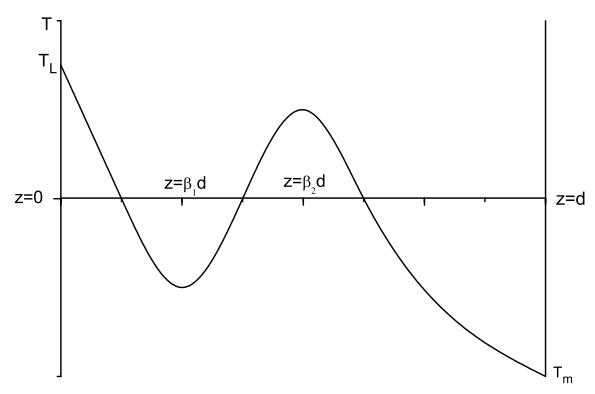

Figure 1. Steady-state temperature profile

Let us denote $\beta=\delta T / d$ and $\delta T=T_{m}-T_{U}$, then, the steady state, for which there is no fluid flow, is given by

$$
\begin{aligned}
& \bar{v}_{i} \equiv 0, \quad \bar{C}=C_{m}-\frac{\Delta C}{d} z, \\
& \bar{T}=-Q_{1} d^{3}\left[\frac{1}{6 d^{3}} z^{3}-\frac{\left(\beta_{1}+\beta_{2}\right)}{4 d^{2}} z^{2}+\frac{\beta_{1} \beta_{2}}{2 d} z\right]+T_{L}, \\
& \frac{d \bar{p}}{d z}=-k_{i} g \rho_{0}\left(1-\alpha_{t}\left(\bar{T}-T_{m}\right)^{2}+\alpha_{c}(\bar{C}-C)\right),
\end{aligned}
$$

where $Q_{1}=\xi_{1} / \kappa_{t}$ and $\Delta C=C_{L}-C_{U}$. To study the stability of (2.1)-(2.4), we introduce a perturbation $\left(u_{i}, \pi, \phi, \theta\right)$ to the steady state solution $\left(\bar{v}_{i}, \bar{p}, \bar{c}, \bar{T}\right)$, by $v_{i}=\bar{v}_{i}+u_{i}, p=\bar{p}+\pi, c=\bar{c}+\phi, T=\bar{T}+\theta$. Using (2.6), the nonlinear perturbation equations have the form

$$
\begin{gathered}
\rho_{0}\left(u_{i, t}+u_{j} u_{i, j}\right)=-\pi_{, i}+\rho_{0} \nu \Delta u_{i}+k_{i} g \rho_{0} \alpha_{t} \theta^{2} \\
+2 k_{i} g \rho_{0} \alpha_{t}\left(\bar{T}-T_{m}\right) \theta-k_{i} g \rho_{0} \alpha_{c} \phi
\end{gathered}
$$




$$
\begin{aligned}
& \theta_{t}+u_{i} \theta_{, i}=-\frac{d \bar{T}}{d z} w+\kappa_{t} \Delta \theta, \\
& \phi_{t}+u_{i} \phi_{, i}=\frac{\Delta C}{d} w+\kappa_{c} \Delta \phi,
\end{aligned}
$$

where $u_{i}$ is solenoidal, i.e. $u_{i, i}=0$.

These equations are conveniently non-dimensionalised with the variables

$$
\begin{aligned}
& \mathbf{x}=\mathbf{x}^{*} d, \quad t=t^{*} \frac{d^{2}}{\nu}, \quad \mathbf{u}=U \mathbf{u}^{*}, \quad \phi=T^{\sharp} \phi^{*}, \quad \pi=P \pi^{*}, \quad U=\frac{\nu}{d}, \\
& P=\frac{\rho_{0} \nu^{2}}{d^{2}}, \quad T_{\phi}^{\sharp}=U \sqrt{\frac{\nu \Delta C}{g \kappa_{c} \alpha_{c} d}}, \quad R_{c}=\sqrt{\frac{g \alpha_{c} d^{3} \Delta C}{\kappa_{c} \nu}}, \quad T_{\theta}^{\sharp}=U \sqrt{\frac{\nu}{g d \kappa_{t} \alpha_{t}}}, \\
& R_{t}=Q_{1} \sqrt{\frac{g d \alpha_{t} d^{9}}{\kappa_{t} \nu}}, \quad P_{s}=\frac{\nu}{\kappa_{c}}, \quad P_{r}=\frac{\nu}{\kappa_{t}} .
\end{aligned}
$$

Here $P_{c}$ and $P_{t}$ are the Prandtl numbers and $R_{t}$ and $R_{c}$ are the thermal and solute Rayleigh numbers. Equations (2.7) in non-dimensional form (dropping stars) become,

$$
\begin{aligned}
& u_{i, t}+u_{j} u_{i, j}=-\pi_{, i}+\Delta u_{i}-2 k_{i} R_{t} f(z) \theta+k_{i} P_{r} \theta^{2}-k_{i} R_{c} \phi, \\
& u_{i, i}=0, \\
& P_{r}\left(\theta_{t}+u_{i} \theta_{, i}\right)=R_{t} f^{\prime}(z) w+\Delta \theta, \\
& P_{s}\left(\phi_{t}+u_{i} \phi_{, i}\right)=R_{c} w+\Delta \phi,
\end{aligned}
$$

where

$$
f(z)=\frac{1}{6}\left(z^{3}-1\right)-\left(\frac{\beta_{1}+\beta_{2}}{4}\right)\left(z^{2}-1\right)+\frac{\beta_{1} \beta_{2}}{2}(z-1) .
$$

The spatial domain is now $\left\{(x, y) \in \mathbb{R}^{2}\right\} \times\{z \in(0,1)\}$. These equations are to be solved together with the boundary conditions

$$
\phi=\theta=u_{i}=0 \quad \text { on } \quad z=0,1,
$$

together with the fact that the $(x, y)$ behaviour of $u_{i}, \theta, \phi, \pi$ satisfies a plane tiling periodic pattern, Chandrasekhar [2], Straughan [19].

\section{$3 \quad$ Linear instability}

In order to study linear instability, we discard the nonlinear terms in (2.8). A time dependence such as $u_{i}=e^{\sigma t} u_{i}(\mathbf{x}), \pi=e^{\sigma t} \pi(\mathbf{x}), \theta=e^{\sigma t} \theta(\mathbf{x}), \phi=e^{\sigma t} \phi(\mathbf{x})$ is now assumed and then, after removing, the pressure perturbation the linearized instability equations that arise from (2.8) are found to be

$$
\begin{aligned}
& \Delta^{2} w-2 R_{t} f(z) \Delta^{*} \theta-R_{c} \Delta^{*} \phi=\sigma \Delta w \\
& R_{t} f^{\prime}(z) w+\Delta \theta=\sigma P_{r} \theta \\
& R_{c} w+\Delta \phi=\sigma P_{s} \phi
\end{aligned}
$$


where $\Delta^{*}$ is the horizontal Laplacian $\Delta^{*}=\partial^{2} / \partial x^{2}+\partial^{2} / \partial y^{2}, D=d / d z$, and (3.1) hold on $\mathbb{R}^{2} \times(0,1)$. To proceed from equations (3.1), a plane tiling form $h(x, y)$ is introduced, (see e.g. [2]), and then we put $w=W(z) h(x, y)$, $\theta=\Theta(z) h(x, y)$ and $\phi=\Phi(z) h(x, y)$ and introduce the wavenumber $a$ by $\Delta^{*} h=-a^{2} h$. Equations (3.1) then yield the eigenvalue problem

$$
\begin{aligned}
& \left(D^{2}-a^{2}\right)^{2} W+2 a^{2} R_{t} f(z) \Theta+a^{2} R_{c} \Phi=\sigma\left(D^{2}-a^{2}\right) W \\
& \left(D^{2}-a^{2}\right) \Theta+R_{t} f^{\prime}(z) W=\sigma P_{r} \Theta \\
& \left(D^{2}-a^{2}\right) \Phi+R_{c} W=\sigma P_{s} \Phi .
\end{aligned}
$$

In $(3.2), z \in(0,1)$, and the boundary conditions are

$$
\Theta=\Phi=W=D W=0, \quad z=0,1 .
$$

Detailed numerical results are presented in Section 6.

Although the linear analysis has been complete numerically, it is possible to use analytic methods to provide a general idea about the stationary and oscillatory neutral lines. In the linear instability analysis, the values of Prandtl numbers play a crucial role in determine where the linear curve is an oscillatory curve or stationary curve. Thus, it is useful to obtain an analytic solution for the problem. Before we start with analytic analysis we suppose that the boundary conditions are two free boundaries i.e. we will solve analytically system (3.2) with respect to the following boundary conditions:

$$
\Theta=\Phi=W=D^{2} W=0, \quad z=0,1 .
$$

The main problem when dealing with (3.2) and (3.4) analytically is the $f(z)$ terms, as they vary over the $z \in[0,1]$ range. However, by taking the average of both polynomials over this range we can proceed analytically so that

$$
\begin{aligned}
f_{a v} & =\int_{0}^{1}\left(\frac{1}{6}\left(z^{3}-1\right)-\frac{\beta_{1}+\beta_{2}}{4}\left(z^{2}-1\right)+\frac{\beta_{1} \beta_{2}}{2}(z-1)\right) d z \\
& =-\left(\frac{1}{8}-\frac{\beta_{1}+\beta_{2}}{6}+\frac{\beta_{1} \beta_{2}}{4}\right)=-\mathcal{A}, \\
f_{a v}^{\prime} & =\int_{0}^{1}\left(\frac{1}{2} z^{2}-\frac{\beta_{1} \beta_{2}}{2} z+\frac{\beta_{1} \beta_{2}}{2}\right) d z=\left(\frac{1}{6}-\frac{\beta_{1}+\beta_{2}}{4}+\frac{\beta_{1} \beta_{2}}{2}\right)=\mathcal{B} .
\end{aligned}
$$

Now, according to the above assumptions, our system can simplified to the following form

$$
\begin{aligned}
& \sigma\left(D^{2}-a^{2}\right) W=\left(D^{2}-a^{2}\right)^{2} W-2 a^{2} R_{t} \mathcal{A} \Theta+a^{2} R_{c} \Phi, \\
& \sigma P_{r} \Theta=\left(D^{2}-a^{2}\right) \Theta+R_{t} \mathcal{B} W, \\
& \sigma P_{s} \Phi=\left(D^{2}-a^{2}\right) \Phi+R_{c} W .
\end{aligned}
$$

Hence, letting $\mathfrak{L}_{1}=\left(D^{2}-a^{2}\right)-\sigma P_{r}$ and $\mathfrak{L}_{2}=\left(D^{2}-a^{2}\right)-\sigma P_{s}$, thus, from $(3.5)_{2}$ and $(3.5)_{3}$ we have

$$
\begin{aligned}
& \mathfrak{L}_{1} \Theta=\left(D^{2}-a^{2}\right) \Theta-\sigma P_{r} \Theta=-R_{t} \mathcal{B} W \\
& \mathfrak{L}_{2} \Phi=\left(D^{2}-a^{2}\right) \Phi-\sigma P_{r} \Phi=-R_{c} W .
\end{aligned}
$$


Now, re-applying $\mathfrak{L}_{1}$ and $\mathfrak{L}_{2}$ to $(3.5)_{1}$ to get

$$
\sigma \mathfrak{L}_{1} \mathfrak{L}_{2}\left(D^{2}-a^{2}\right) W=\mathfrak{L}_{1} \mathfrak{L}_{2}\left(D^{2}-a^{2}\right)^{2} W+2 \mathcal{A B} a^{2} R_{t}^{2} \mathfrak{L}_{2}(W)-a^{2} R_{c}^{2} \mathfrak{L}_{1}(W) .
$$

After make simple calculation we have

$$
\begin{aligned}
\sigma\left(D^{2}\right. & \left.-a^{2}-\sigma P_{s}\right)\left(D^{2}-a^{2}-\sigma P_{r}\right)\left(D^{2}-a^{2}\right) W \\
& =\left(D^{2}-a^{2}-\sigma P_{s}\right)\left(D^{2}-a^{2}-\sigma P_{r}\right)\left(D^{2}-a^{2}\right)^{2} W \\
& +2 \mathcal{A B} a^{2} R_{t}^{2}\left(D^{2}-a^{2}-\sigma P_{s}\right) W-a^{2} R_{c}^{2}\left(D^{2}-a^{2}-\sigma P_{r}\right) W .
\end{aligned}
$$

Because of the boundary conditions $W=0, D^{2} W=0$ on $z=0,1$ then $W$ can be expanded as a sine series of terms like $\sin (n \pi z)$. Then, with $\Lambda=n^{2} \pi^{2}+a^{2}$, where $a$ being a wavenumber, from system (3.6) we derive

$$
\begin{gathered}
-\sigma \Lambda^{3}-\sigma^{2}\left(P_{r}+P_{s}\right) \Lambda^{2}-\sigma^{3} \Lambda P_{r} P_{s}=\Lambda^{4}+\sigma\left(P_{r}+P_{s}\right) \Lambda^{3}+\sigma^{2} \Lambda^{2} P_{r} P_{s} \\
+a^{2}\left(R_{c}^{2}-2 \mathcal{A B} R_{t}^{2}\right) \Lambda+a^{2}\left(P_{r} R_{c}^{2}-2 \mathcal{A B} P_{s} R_{t}^{2}\right) \sigma .
\end{gathered}
$$

The stationary convection curve $(\sigma=0)$ is then given by

$$
R_{t}^{2}=\frac{1}{2 \mathcal{A B}}\left(\frac{\Lambda^{3}}{a^{2}}+R_{c}^{2}\right) .
$$

Now, differentiating (3.8) with respect to $n^{2}$ we have

$$
\frac{\partial R_{t}^{2}}{\partial n^{2}}=\frac{1}{2 \mathcal{A B}}\left(\frac{3\left(n^{2} \pi^{2}+a^{2}\right)^{2} \pi^{2}}{a^{2}}\right)>0,
$$

so we can take $n=1$, and hence

$$
R_{t}^{2}=\frac{1}{2 \mathcal{A B}}\left(\frac{\left(\pi^{2}+a^{2}\right)^{3}}{a^{2}}+R_{c}^{2}\right) .
$$

Then we minimize (3.9) with respect to $a^{2}$ thus we have $a_{c}^{2}=\pi^{2} / 2$. Substituting this $a^{2}$ value into (3.9) we can evaluate

$$
R a_{L}=\frac{1}{2 \mathcal{A B}}\left(\frac{27}{4} \pi^{4}+R_{c}^{2}\right) .
$$

For the general case (3.7) we put $\left(\sigma=\sigma_{r}+i \sigma_{i}\right)$ and the instability boundary is found when $\sigma_{r}=0$. Thus, we follow the method of Chandrasekhar [2], P.114, and put $\sigma=i \sigma_{i}$ in (3.7). Taking real and imaginary parts of the resulting equation and then eliminating $\sigma_{i}^{2}$ we derive the equation for overstability,

$$
R_{t}^{2}=\frac{1}{2 \mathcal{A B}}\left(\frac{\Lambda^{3}}{a^{2}} \frac{\left(1-P_{1} P_{2}\right)}{\left(1-P_{s} P_{1}\right)}+R_{c}^{2} \frac{\left(1-P_{r} P_{1}\right)}{\left(1-P_{s} P_{1}\right)}\right),
$$

where $P_{1}=\left(\left(P_{r}+P_{s}\right) / P_{r} P_{s}\right)+1$ and $P_{2}=P_{r}+P_{s}+1$. It is simple to show that $\frac{\partial R_{t}^{2}}{\partial n^{2}}>0$, so that $n=1$ can be taken. Now we minimize (3.10) with respect to $a^{2}$ we find that $a_{c}^{2}=\pi^{2} / 2$ and

$$
R a_{L}=\frac{1}{2 \mathcal{A B}}\left(\frac{27 \pi^{4}}{4} \frac{\left(1-P_{1} P_{2}\right)}{\left(1-P_{s} P_{1}\right)}+R_{c}^{2} \frac{\left(1-P_{r} P_{1}\right)}{\left(1-P_{s} P_{1}\right)}\right) .
$$




\section{Nonlinear energy stability theory}

When adopting a linear analysis approach, the perturbation to the steady state is assumed to be small, and so nonlinear terms in the governing set of partial differential equations are discarded. It has been proved that linear analysis often provides little information on the behavior of the nonlinear system [19], so in such cases only instability can be deduced from the linear thresholds, as any potential growth in the nonlinear terms is not considered.

\subsection{Weighted energy method}

Let $V$ be a period cell for a disturbance to $(2.8)$, and let $\|\cdot\|$ and $(\cdot, \cdot)$ be the norm and inner product on $L^{2}(V)$. Next, multiply $(2.8)_{1},(2.8)_{3}$ and $(2.8)_{4}$ by $u_{i}, \theta$ and $\phi$ and integrating over $V$, a cubic nonlinearity $\left(w, \theta^{2}\right)$ is obtained from $(2.8)_{1}$. To remove the cubic term and hence achieve a global nonlinear stability result, we use a weighted energy technique. First multiply $(2.8)_{1}$ by $u_{i}$ and integrate over $V$ to obtain

$$
\frac{d}{d t}\left(\frac{1}{2}\|u\|^{2}\right)=-\|\nabla u\|-2 R_{t}(f(z) w, \theta)+P_{r}\left(w, \theta^{2}\right)-R_{c}(w, \phi) .
$$

A weight function is then introduced $\hat{\mu}=\mu-2 z$, where $\mu>2$ is some constant coupling parameter chosen so that $\hat{\mu}>0$. Using $(2.8)_{3}$ can be deduced that

$$
\frac{d}{d t}\left(\frac{P_{r}}{2} \int_{V} \hat{\mu} \theta^{2} d V\right)=-P_{r}\left(w, \theta^{2}\right)-\int_{V} \hat{\mu} \nabla \theta \nabla \theta d V+R_{t}\left(\hat{\mu} w, \theta f^{\prime}(z)\right) .
$$

Finally, multiplying $(2.8)_{4}$ by $\phi$ and integrating over $V$

$$
\frac{d}{d t}\left(\frac{P_{s}}{2}\|\phi\|^{2}\right)=-\|\nabla \phi\|^{2}+R_{c}(w, \phi) .
$$

Then,

$$
\frac{d E}{d t}=\mathcal{I}-\mathcal{D}
$$

where the functions $E$ and $I$ are given by

$$
\begin{aligned}
& E(t)=\frac{1}{2}\|\mathbf{u}\|^{2}+\frac{P_{r}}{2} \int \hat{\mu} \theta^{2} d V+\frac{\lambda P_{s}}{2}\|\phi\|^{2} \\
& \mathcal{I}=-2 R_{t}(f(z) w, \theta)-R_{c}(w, \phi)+R_{t}\left(f^{\prime}(z) \hat{\mu} w, \theta\right)+\frac{R_{c} \lambda}{2}(w, \phi)
\end{aligned}
$$

with the dissipation $D$ being defined by

$$
\mathcal{D}=\lambda\|\nabla \phi\|^{2}+\|\nabla \mathbf{u}\|^{2}+\int \hat{\mu} \nabla \theta \nabla \theta d V
$$

where $\mathbf{u}$ is explicitly written as $\mathbf{u}=(u, v, w)$. Define $R_{E}$ by

$$
\frac{1}{R_{E}}=\max _{\mathcal{H}} \frac{\mathcal{I}}{\mathcal{D}}
$$


where $H$ is the space of admissible functions, i.e. $u_{i}, \theta, \phi \in H^{1}(V)$ with $u_{i}$ solenoidal and $u_{i}, \theta, \phi$ satisfying the boundary conditions. Then from (4.1) we derive

$$
\frac{d E}{d t} \leq-\mathcal{D}\left(1-\frac{1}{R_{E}}\right)
$$

Poincare's inequality ensures that there is a constant $c>0$ such that $\mathcal{D}>$ $c E$ and then if $R_{E}>1$, one may show from inequality (4.6) that $E$ decays exponentially and we have global nonlinear stability, i.e. for all initial data. The nonlinear stability threshold then requires the solution of (4.5). The EulerLagrange equations which arise from (4.5) are

$$
\begin{aligned}
& k_{i} R_{t}\left(\hat{\mu} f^{\prime}(z)-2 f(z)\right) \theta+k_{i} R_{c}(\lambda-1) \phi+2 \Delta u=\iota_{, i}, \\
& R_{t}\left(\hat{\mu} f^{\prime}(z)-2 f(z)\right) w+2 \hat{\mu} \Delta \theta-4 \frac{d \theta}{d z}=0, \\
& R_{c}(\lambda-1) w+2 \lambda \Delta \phi=0
\end{aligned}
$$

where $\iota$ is a Lagrange multiplier. To remove the Lagrange multiplier we take the third component of the double curl of $(4.4)_{1}$, and introducing the normal mode representation and notation as presented in Section 3, thus (4.2) then becomes

$$
\begin{aligned}
& 2\left(D^{2}-a^{2}\right)^{2} W-a^{2} R_{c}(\lambda-1) \Phi=a^{2} R_{t}\left(\hat{\mu} f^{\prime}(z)-2 f(z)\right) \Theta, \\
& 2 \hat{\mu}\left(D^{2}-a^{2}\right) \Theta-4 D z=-R_{t}\left(\hat{\mu} f^{\prime}(z)-2 f(z)\right) W, \\
& 2 \lambda\left(D^{2}-a^{2}\right) \Phi+R_{c}(\lambda-1) W=0,
\end{aligned}
$$

together with boundary conditions (3.3).

\subsection{A generalized energy method}

The theory of generalized energy stability is very useful since it is often possible to derive very sharp nonlinear stability thresholds by an appropriate choice of Lyapunov function. However, the real virtue of a nonlinear energy analysis is when it can be employed to yield stability thresholds which are valid for all initial data, or at least for a large set of initial data. As Straughan ( [17], p. 157) remarks, one of the key current objectives of nonlinear energy-stability theory is to be applicable for all initial data and so derive an unconditional stability result, but simultaneously to maintain the nonlinear stability Rayleigh number threshold (or other suitable non-dimensional parameter threshold) such that it is physically useful in mundane situations.

One may argue whether the form of the NavierStokes equations given in (2.1) is actually adequate for a nonlinear analysis. Indeed, Straughan [18] adapts several models of Ladyzhenskaya to be applicable to thermal convection. In addition, a recent study of Bulíček et al. [1] likewise argues for a nonlinear dependence of stress upon the symmetric part of the velocity gradient. Hence, we now consider a modification of (2.1), which is consistent with model III of Ladyzhenskaya, as described by Straughan [18], but adapted to the penetrative convection problem in hand. Thus, we consider equation (2.1) modified to

$$
\rho_{0}\left(v_{i, t}+v_{j} v_{i, j}\right)=-p_{, i}+\rho_{0}\left[\left[\nu+\hat{\nu}_{1}|\nabla \mathbf{v}|\right] v_{i},_{j}\right],_{j}-\rho_{0} k_{i} g \rho(T, C),
$$


where $\hat{\nu}_{1}$ is another positive coefficient introduced by Ladyzhenskaya $[11,12,13]$. Equations (2.2)-(2.5) still hold as does the steady solution and the linearized analysis. By adopting equation (4.9), one now derives, instead of the nonlinear perturbation equations (2.8), the system

$$
\begin{aligned}
& u_{i, t}+u_{j} u_{i, j}=-\pi_{, i}+\Delta u_{i}+\nu_{1} \nabla|\nabla u|^{2}-2 k_{i} R_{t} f(z) \theta+k_{i} P_{r} \theta^{2}-k_{i} R_{c} \phi, \\
& u_{i, i}=0 \\
& P_{r}\left(\theta_{t}+u_{i} \theta_{, i}\right)=R_{t} f^{\prime}(z) w+\Delta \theta \\
& P_{s}\left(\phi_{t}+u_{i} \phi_{, i}\right)=R_{c} w+\Delta \phi
\end{aligned}
$$

where $\nu_{1}$ is a non-dimensional form of $\hat{\nu}_{1}$. The boundary conditions are as in (2.9). To develop a nonlinear stability analysis, we construct the identities

$$
\begin{aligned}
& \frac{1}{2} \frac{d}{d t}\|\mathbf{u}\|^{2}=-\|\nabla \mathbf{u}\|^{2}-2 R_{t}(w, f(z) \theta)+P_{r}\left(w, \theta^{2}\right)-R_{c}(w, \phi)-\nu_{1} \int_{V}|\nabla u|^{3} d x, \\
& \frac{P_{r}}{2} \frac{d}{d t}\|\theta\|^{2}=R_{t}\left(f^{\prime}(z) w, \theta\right)-\|\nabla \theta\|^{2} \\
& \frac{P_{s}}{2} \frac{d}{d t}\|\phi\|^{2}=R_{c}(w, \phi)-\|\nabla \phi\|^{2} .
\end{aligned}
$$

To proceed, we derive an inequality involving the $L^{3}(V)$ norm of $\theta$, thus, we find

$$
\frac{P_{r}}{3} \frac{d}{d t}\|\theta\|_{3}^{3}=R \int_{V} f^{\prime}(z) w \theta^{2}(\operatorname{sgn} \theta) d x-\frac{8}{9}\left\|\nabla|\theta|^{3 / 2}\right\|^{2} .
$$

With the aid of Poincarés inequality we then deduce

$$
\frac{P_{r}}{3} \frac{d}{d t}\|\theta\|_{3}^{3} \leq R \int_{V} f^{\prime}(z) w \theta^{2}(\operatorname{sgn} \theta) d x-\frac{8}{9} \pi^{2}\|\theta\|_{3}^{3} .
$$

Next, we see from Poincarés inequality that

$$
\|w\|_{3}^{3} \leq \frac{1}{\pi^{2}}\left\|\nabla|w|^{3 / 2}\right\|^{2}=\frac{9}{4 \pi^{2}} \int_{V}|w||\nabla w|^{2} d x \leq \frac{9}{4 \pi^{2}}\|w\|_{3}\|\nabla w\|_{3}^{2} .
$$

Thus,

$$
\|w\|_{3}^{3} \leq \frac{27}{8 \pi^{3}}\|\nabla w\|_{3}^{3}
$$

We now use Youngs inequality for positive $\alpha$ to obtain

$$
\begin{aligned}
& R \int_{V} f^{\prime}(z) w \theta^{2}(\operatorname{sgn} \theta) d x \leq R f_{m}^{\prime}\left(\frac{1}{3 \alpha^{3}}\|w\|_{3}^{3}+\frac{2 \alpha^{3 / 2}}{3}\|\theta\|_{3}^{3}\right), \\
& P_{r}\left(w, \theta^{2}\right) \leq P_{r}\left(\frac{1}{3 \alpha^{3}}\|w\|_{3}^{3}+\frac{2 \alpha^{3 / 2}}{3}\|\theta\|_{3}^{3}\right)
\end{aligned}
$$

where $f_{m}^{\prime}$ denotes the maximum of $f_{m}^{\prime}(z)$ on $(0,1)$. Upon using equations (4.11)-(4.16), we may derive

$$
\begin{aligned}
\frac{d \mathcal{E}}{d t}= & \mathcal{I}^{*}-\mathcal{D}^{*}-\left(\frac{8 \nu_{1} \pi^{3}}{27}-\frac{1}{3 \alpha^{3}}\left[P_{r}+R \hat{a} f_{m}^{\prime}\right]\right)\|w\|_{3}^{3} \\
& -\left(\frac{8 \hat{a} \pi^{2}}{9}-\frac{2 \alpha^{3 / 2}}{3}\left[P_{r}+R \hat{a} f_{m}^{\prime}\right]\right)\|\theta\|_{3}^{3}
\end{aligned}
$$


where $\hat{a}>0$ is a constant to be chosen and $\mathcal{E}, \mathcal{I}^{*}$ and $\mathcal{D}^{*}$ are defined by

$$
\begin{aligned}
& \mathcal{E}=\frac{1}{2}\|\mathbf{u}\|^{2}+P_{r} \frac{\lambda_{1}}{2}\|\theta\|^{2}+P_{s} \frac{\lambda_{2}}{2}\|\phi\|^{2}+\frac{\hat{a} P_{r}}{3}\|\theta\|_{3}^{3}, \\
& \mathcal{I}^{*}=R_{t}\left(w, \theta\left[-2 f(z)+\lambda_{1} f^{\prime}(z)\right]\right)-R_{c}\left(w, \phi\left[1-\lambda_{2}\right]\right), \\
& \mathcal{D}^{*}=\|\nabla \mathbf{u}\|^{2}+\lambda_{1}\|\nabla \theta\|^{2}+\lambda_{2}\|\nabla \phi\|^{2},
\end{aligned}
$$

where $\lambda_{1}>0$ and $\lambda_{2}>0$ be constants to be selected optimally. To progress beyond inequality (4.17), we require

$$
\frac{8 \nu_{1} \pi^{3}}{27}-\frac{1}{3 \alpha^{3}}\left[P_{r}+R \hat{a} f_{m}^{\prime}\right]=0 \text { and } \frac{8 \hat{a} \pi^{2}}{9}-\frac{2 \alpha^{3 / 2}}{3}\left[P_{r}+R \hat{a} f_{m}^{\prime}\right]>0 .
$$

Choose now $\alpha$ such that

$$
\alpha^{3}=\frac{9\left(P_{r}+R \hat{a} f_{m}^{\prime}\right)}{8 \pi^{3} \nu_{1}}
$$

Then from $(4.19)_{2}$, we may obtain

$$
\sqrt{\nu_{1}}>\frac{9\left(P_{r}+R \hat{a} f_{m}^{\prime}\right)^{3 / 2}}{8 \sqrt{2} \pi^{7 / 2} \hat{a}} .
$$

Optimize the right-hand side of (4.20) to obtain $\hat{a}=2 \operatorname{Pr} / R f_{m}^{\prime}$. This then leads to the restriction on $\nu_{1}$,

$$
\nu_{1}>\frac{2187}{512 \pi^{7}} P_{r} R^{2} f_{m}^{\prime 2}
$$

With the above choices, the coefficient of $\|\theta\|_{3}^{3}$ in (4.17) becomes

$$
\frac{8 \hat{a} \pi^{2}}{9}-\frac{2 \alpha^{3 / 2}}{3}\left[P_{r}+R \hat{a} f_{m}^{\prime}\right]=\hat{\kappa}
$$

Then from inequality (4.22), we may find

$$
\frac{d \mathcal{E}}{d t} \leq \mathcal{I}^{*}-\mathcal{D}^{*}-\hat{\kappa}\|\theta\|_{3}^{3} \leq-\mathcal{D}^{*}\left(1-\frac{1}{R_{E}}\right)-\hat{\kappa}\|\theta\|_{3}^{3},
$$

where

$$
\frac{1}{R_{E}}=\max _{\mathcal{H}} \frac{\mathcal{I}^{*}}{\mathcal{D}^{*}}
$$

where $\mathcal{H}$ is the space of admissible solutions. Then, collecting together terms, one finds

$$
\mathcal{E}=\frac{1}{2}\|\mathbf{u}\|^{2}+P_{r} \frac{\lambda_{1}}{2}\|\theta\|^{2}+P_{s} \frac{\lambda_{2}}{2}\|\phi\|^{2}+\frac{2 P_{r}^{2}}{3 R_{t} f_{m}^{\prime}}\|\theta\|_{3}^{3},
$$

and then with use of Poincarés inequality

$$
\mathcal{D}^{*} \geq \pi^{2}\|\mathbf{u}\|^{2}+\lambda_{1} \pi^{2}\|\theta\|^{2}+\lambda_{2} \pi^{2}\|\phi\|^{2} .
$$


Put $\hat{c}=1-R / R_{E}$ and then from (4.23), if $R<R_{E}$,

$$
\frac{d \mathcal{E}}{d t} \leq-\hat{c} \pi^{2}\left(\|\mathbf{u}\|^{2}+\lambda_{1}\|\theta\|^{2}+\lambda_{2}\|\phi\|^{2}\right)-\hat{\kappa}\|\theta\|_{3}^{3} .
$$

Exponential decay of $\mathcal{E}$ may be deduced from (4.25) for all initial data, provided $\nu_{1}$ satisfies condition (4.21), and thus global nonlinear stability holds when $R<R_{E}$.

The Euler-Lagrange equations which arise from (4.24) yield an eigenvalue problem for $R_{t}$, and these are found to be

$$
\begin{aligned}
& 2 \Delta u_{i}+R_{t} k_{i}\left(-2 f(z)+\lambda_{1} f^{\prime}(z)\right) \theta-R_{c} k_{i}\left(1-\lambda_{2}\right) \phi=\zeta_{, i}, \\
& 2 \lambda_{1} \Delta \theta+R_{t}\left(-2 f_{1}(z)+\lambda_{1} f^{\prime}(z)\right) w=0, \\
& 2 \lambda_{2} \Delta \phi-R_{c}\left(1-\lambda_{2}\right) w=0 .
\end{aligned}
$$

The Lagrange multiplier $\zeta$ is removed from (4.26) by taking curl curl and keeping the third component of the result. This leads to the system

$$
-2 \Delta^{2} w-R_{t}\left(-2 f(z)+\lambda_{1} f^{\prime}(z)\right) \Delta^{*} \theta+R_{c}\left(1-\lambda_{2}\right) \Delta^{*} \phi=0 .
$$

The functions $w, \theta$ and $\phi$ are written as $w=W(z) h(x, y), \theta=\Theta h(x, y)$ and $\phi=\Phi(z) h(x, y)$, and this results in having to solve the following eigenvalue problem with

$$
\begin{aligned}
& 2\left(D^{2}-a^{2}\right)^{2} W+a^{2} R_{c}\left(1-\lambda_{2}\right) \Phi=a^{2} R_{t}\left(-2 f(z)+\lambda_{1} f^{\prime}(z)\right) \Theta, \\
& 2 \lambda_{1}\left(D^{2}-a^{2}\right) \Theta=-R_{t}\left(-2 f(z)+\lambda_{1} f^{\prime}(z)\right) W \\
& 2 \lambda_{2}\left(D^{2}-a^{2}\right) \Phi-R_{c}\left(1-\lambda_{2}\right) W=0 .
\end{aligned}
$$

The boundary conditions that apply to $W, \Theta$ and $\Phi$ are as in (3.3).

\section{Numerical technique}

In this section, we use the Chebyshev collocation method to solve the eigenvalue systems (3.2), (4.8) and (4.27). In the Chebyshev collocation method, system (3.2) is rewritten in terms of 2 nd order derivatives only. Letting $\Pi=D W$ and $\Xi=D^{3} W,(3.2)$ can be expressed as the four 2 nd order equations. The system is then transformed onto the Chebyshev domain $(-1,1)$ and the solutions $W, \theta$ and $\phi$ treated as independent variables and expanded in a series of Chebyshev polynomials

$$
\begin{aligned}
& \Pi=\sum_{n=0}^{N} \Pi_{n} T_{n}(z), \quad W=\sum_{n=0}^{N} w_{n} T_{n}(z), \\
& \Theta=\sum_{n=0}^{N} \Theta_{n} T_{n}(z), \quad \Phi=\sum_{n=0}^{N} \Phi_{n} T_{n}(z),
\end{aligned}
$$

then, we insert (5.1) into the equations (3.2), and then substitute the GaussLabatto points which are defined by

$$
y_{i}=\cos \left(\frac{\pi i}{N-3}\right), \quad i=0, \ldots, N-2 .
$$


Thus, we obtain $5 N-5$ algebraic equations for $5 N+5$ unknowns $W_{0}, \ldots, W_{N}$, $\Pi_{0}, \ldots, \Pi_{N}, \Theta_{0}, \ldots, \Theta_{N}, \Phi_{0}, \ldots, \Phi_{N}$. Now, we can add ten rows using the boundary conditions (3.3) as follows

$$
\begin{aligned}
& B C_{1}: \sum_{n=0}^{N} W_{n}=0, \quad B C_{2}: \sum_{n=0}^{N}(-1)^{n} W_{n}=0, \quad B C_{3}: \sum_{n=0}^{N} \Pi_{n}=0, \\
& B C_{4}: \sum_{n=0}^{N}(-1)^{n} \Pi_{n}=0, \quad B C_{5}: \sum_{n=0}^{N} \Theta_{n}=0, \quad B C_{6}: \sum_{n=0}^{N}(-1)^{n} \Theta_{n}=0, \\
& B C_{7}: \sum_{n=0}^{N} \Phi_{n}=0, \quad B C_{8}: \sum_{n=0}^{N}(-1)^{n} \Phi_{n}=0 .
\end{aligned}
$$

The inner product of each equation is taken with some $T_{k}$ and the orthogonality of the Chebyshev polynomials exploited to obtain the following generalised eigenvalue problem:

$$
\left(\begin{array}{cccc}
2 D & -I & O & O \\
B C_{1} & 0 \ldots 0 & 0 \ldots 0 & 0 \ldots 0 \\
B C_{2} & 0 \ldots 0 & 0 \ldots 0 & 0 \ldots 0 \\
\Omega_{1} & \Omega_{2} & R_{t} \Upsilon & a^{2} R_{c} I \\
0 \ldots 0 & B C_{3} & 0 \ldots 0 & 0 \ldots 0 \\
0 \ldots 0 & B C_{4} & 0 \ldots 0 & 0 \ldots 0 \\
R_{t} \Sigma & O & \Omega_{3} & O \\
0 \ldots 0 & 0 \ldots 0 & B C_{5} & 0 \ldots 0 \\
0 \ldots 0 & 0 \ldots 0 & B C_{6} & 0 \ldots 0 \\
R_{c} I & O & O & \Omega_{3} \\
0 \ldots 0 & 0 \ldots 0 & B C_{7} & 0 \ldots 0 \\
0 \ldots 0 & 0 \ldots 0 & B C_{8} & 0 \ldots 0
\end{array}\right) X=\sigma\left(\begin{array}{cccc}
O & O & O & O \\
0 \ldots 0 & 0 \ldots 0 & 0 \ldots 0 & 0 \ldots 0 \\
0 \ldots 0 & 0 \ldots 0 & 0 \ldots 0 & 0 \ldots 0 \\
-a^{2} I & 2 D & O & O \\
0 \ldots 0 & 0 \ldots 0 & 0 \ldots 0 & 0 \ldots 0 \\
0 \ldots 0 & 0 \ldots 0 & 0 \ldots 0 & 0 \ldots 0 \\
O & O & P_{r} I & O \\
0 \ldots 0 & 0 \ldots 0 & 0 \ldots 0 & 0 \ldots 0 \\
0 \ldots 0 & 0 \ldots 0 & 0 \ldots 0 & 0 \ldots 0 \\
O & O & O & P_{s} I \\
0 \ldots 0 & 0 \ldots 0 & 0 \ldots 0 & 0 \ldots 0 \\
0 \ldots 0 & 0 \ldots 0 & 0 \ldots 0 & 0 \ldots 0
\end{array}\right) X,
$$

where $X=\left(W_{0}, \ldots, W_{N}, \Pi_{0}, \ldots, \Pi_{N}, \Xi_{0}, \ldots, \Xi_{N}, \Theta_{0}, \ldots, \Theta_{N}, \Phi_{0}, \ldots, \Phi_{N}\right), O$ is the zeros matrix,

$$
\begin{aligned}
& I\left(n_{1}, n_{2}\right)=T_{n_{2}}\left(z_{n_{1}}\right), \quad D\left(n_{1}, n_{2}\right)=T_{n_{2}}^{\prime}\left(z_{n_{1}}\right), \quad D^{2}\left(n_{1}, n_{2}\right)=T_{n_{2}}^{\prime \prime}\left(z_{n_{1}}\right), \\
& D^{3}\left(n_{1}, n_{2}\right)=T_{n_{2}}^{\prime \prime \prime}\left(z_{n_{1}}\right), \quad \Omega_{1}\left(n_{1}, n_{2}\right)=a^{4} I\left(n_{1}, n_{2}\right), \\
& \Omega_{2}\left(n_{1}, n_{2}\right)=8 D^{3}\left(n_{1}, n_{2}\right)-4 a^{2} D\left(n_{1}, n_{2}\right), \\
& \Omega_{3}\left(n_{1}, n_{2}\right)=4 D^{2}\left(n_{1}, n_{2}\right)-a^{2} I\left(n_{1}, n_{2}\right), \\
& \Upsilon\left(n_{1}, n_{2}\right)=2 a^{2} f\left(z_{n_{1}}\right) I\left(n_{1}, n_{2}\right), \\
& \Sigma\left(n_{1}, n_{2}\right)=f^{\prime}\left(z_{n_{1}}\right) I\left(n_{1}, n_{2}\right), \quad n_{1}=0, \ldots, N-2, \quad n_{2}=0, \ldots, N .
\end{aligned}
$$

We computed the differentiation matrices, which are corresponded to the trail functions (5.1) analytically using Matlab routines.

Returning to the nonlinear eigenvalue system of the weighted energy method 
(4.8), the the Chebyshev collocation method yields

$\left(\begin{array}{cccc}2 D & -I & O & O \\ B C_{1} & 0 \ldots 0 & 0 \ldots 0 & 0 \ldots 0 \\ B C_{2} & 0 \ldots 0 & 0 \ldots 0 & 0 \ldots 0 \\ \Omega_{1} & \Omega_{2} & O & -\frac{a^{2}}{2} R_{c} \Lambda_{2} \\ 0 \ldots 0 & B C_{3} & 0 \ldots 0 & 0 \ldots 0 \\ 0 \ldots 0 & B C_{4} & 0 \ldots 0 & 0 \ldots 0 \\ O & O & \Omega_{4} & O \\ 0 \ldots 0 & 0 \ldots 0 & B C_{5} & 0 \ldots 0 \\ 0 \ldots 0 & 0 \ldots 0 & B C_{6} & 0 \ldots 0 \\ \Lambda_{2} & O & O & 2 \lambda \Omega_{3} \\ 0 \ldots 0 & 0 \ldots 0 & 0 \ldots 0 & B C_{7} \\ 0 \ldots 0 & 0 \ldots 0 & 0 \ldots 0 & B C_{8}\end{array}\right) X=R_{t}\left(\begin{array}{cccc}O & O & O & O \\ 0 \ldots 0 & 0 \ldots 0 & 0 \ldots 0 & 0 \ldots 0 \\ 0 \ldots 0 & 0 \ldots 0 & 0 \ldots 0 & 0 \ldots 0 \\ O & O & \frac{a^{2}}{2} \Lambda_{1} & O \\ 0 \ldots 0 & 0 \ldots 0 & 0 \ldots 0 & 0 \ldots 0 \\ 0 \ldots 0 & 0 \ldots 0 & 0 \ldots 0 & 0 \ldots 0 \\ -\Lambda_{1} & O & O & O \\ 0 \ldots 0 & 0 \ldots 0 & 0 \ldots 0 & 0 \ldots 0 \\ 0 \ldots 0 & 0 \ldots 0 & 0 \ldots 0 & 0 \ldots 0 \\ O & O & O & O \\ 0 \ldots 0 & 0 \ldots 0 & 0 \ldots 0 & 0 \ldots 0 \\ 0 \ldots 0 & 0 \ldots 0 & 0 \ldots 0 & 0 \ldots 0\end{array}\right) X$

where

$$
\begin{aligned}
& \left.\left.\Omega_{4} n_{1}, n_{2}\right)=2 \hat{\mu}\left(z_{n_{1}}\right) \Omega_{3} n_{1}, n_{2}\right)-8 D\left(n_{1}, n_{2}\right), \\
& \Lambda_{1}\left(n_{1}, n_{2}\right)=\left(\hat{\mu}\left(z_{n_{1}}\right) f^{\prime}\left(z_{n_{1}}\right)-f\left(z_{n_{1}}\right)\right) I\left(n_{1}, n_{2}\right), \\
& \Lambda_{2}\left(n_{1}, n_{2}\right)=R_{c}(\lambda-1) I\left(n_{1}, n_{2}\right) .
\end{aligned}
$$

For the nonlinear eigenvalue system of the generalized energy method (4.27), the the Chebyshev collocation method yields

$$
\left(\begin{array}{cccc}
2 D & -I & O & O \\
B C_{1} & 0 \ldots 0 & 0 \ldots 0 & 0 \ldots 0 \\
B C_{2} & 0 \ldots 0 & 0 \ldots 0 & 0 \ldots 0 \\
\Omega_{1} & \Omega_{2} & O & -\frac{a^{2}}{2} \Lambda_{2} \\
0 \ldots 0 & B C_{3} & 0 \ldots 0 & 0 \ldots 0 \\
0 \ldots 0 & B C_{4} & 0 \ldots 0 & 0 \ldots 0 \\
O & O & 2 \lambda_{1} \Omega_{3} & O \\
0 \ldots 0 & 0 \ldots 0 & B C_{5} & 0 \ldots 0 \\
0 \ldots 0 & 0 \ldots 0 & B C_{6} & 0 \ldots 0 \\
\Lambda_{2} & O & O & 2 \lambda_{2} \Omega_{3} \\
0 \ldots 0 & 0 \ldots 0 & 0 \ldots 0 & B C_{7} \\
0 \ldots 0 & 0 \ldots 0 & 0 \ldots 0 & B C_{8}
\end{array}\right) X=R_{t}\left(\begin{array}{cccc}
O & O & O & O \\
0 \ldots 0 & 0 \ldots 0 & 0 \ldots 0 & 0 \ldots 0 \\
0 \ldots 0 & 0 \ldots 0 & 0 \ldots 0 & 0 \ldots 0 \\
O & O & \frac{a^{2}}{2} \Lambda_{1} & O \\
0 \ldots 0 & 0 \ldots 0 & 0 \ldots 0 & 0 \ldots 0 \\
0 \ldots 0 & 0 \ldots 0 & 0 \ldots 0 & 0 \ldots 0 \\
-\Lambda_{1} & O & O & O \\
0 \ldots 0 & 0 \ldots 0 & 0 \ldots 0 & 0 \ldots 0 \\
0 \ldots 0 & 0 \ldots 0 & 0 \ldots 0 & 0 \ldots 0 \\
O & O & O & O \\
0 \ldots 0 & 0 \ldots 0 & 0 \ldots 0 & 0 \ldots 0 \\
0 \ldots 0 & 0 \ldots 0 & 0 \ldots 0 & 0 \ldots 0
\end{array}\right) X
$$

where

$$
\begin{aligned}
& \Lambda_{1}\left(n_{1}, n_{2}\right)=\left(\lambda_{1} f^{\prime}\left(z_{n_{1}}\right)-2 f\left(z_{n_{1}}\right)\right) I\left(n_{1}, n_{2}\right) \\
& \Lambda_{2}\left(n_{1}, n_{2}\right)=R_{c}\left(\lambda_{2}-1\right) I\left(n_{1}, n_{2}\right) .
\end{aligned}
$$

\section{$6 \quad$ Stability analysis results}

In this section we report our numerical results of systems (5.2), (5.3) and (5.4). We have solved system (5.2) for eigenvalues $\sigma_{j}$ by using the $Q Z$ algorithm from Matlab routines. Once the eigenvalues $\sigma_{j}$ are found we use the secant 
method to locate where $\sigma_{j}^{R}, \sigma_{j}=\sigma_{j}^{R}+\sigma_{j}^{I}$ being the real and imaginary parts of eigenvalue $\sigma_{j}$. The value of $R$ which makes $\sigma_{1}^{R}=0, \sigma_{1}^{R}$ being the largest eigenvalue, is the critical value of $R$ for $a^{2}$ fixed. We then use golden section search to minimize over $a^{2}$ and find the critical value of $R^{2}$ for linear instability. Numerical results are reported in the next section. In our use of the Chebyshev collocation method, we used polynomial of degree between 20 and 30. Usually 25 was found to be sufficient but convergence was checked by varying the degree by examining the convergence of the associated eigenvector (which yields the approximate associated eigenfunction).

Moreover, systems (5.3) and (5.4) have been solved for eigenvalues $R a_{E}$, then, we can determine the critical Rayleigh $R a_{E}$ for fixed $a^{2}, \lambda_{1}$ and $\lambda_{2}$. Next, we employ golden section search to minimize in $a^{2}$ and then maximize in $\lambda_{1}$ and $\lambda_{2}$ to determine $R a_{E}$ for nonlinear energy stability,

$$
R a_{E}=\max _{\lambda_{1}, \lambda_{2}} \min _{a^{2}} R^{2}\left(a^{2}, \lambda_{1}, \lambda_{2}\right),
$$

where for all $R^{2}<R a_{E}$ we have stability. In fact, the optimization problem (6.1) turns out to be very tricky. Numerically it was found that there are local maxima and one has to be very careful when searching to locate a maximum which is useful.

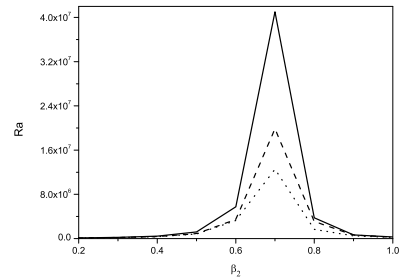

(a) $\beta_{1}=0.1$

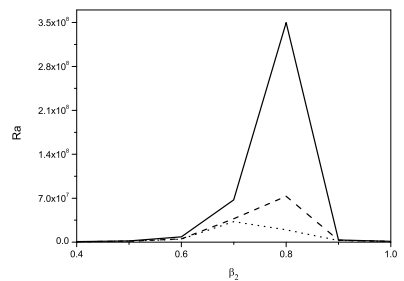

(c) $\beta_{1}=0.3$

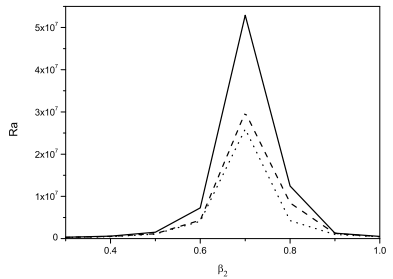

(b) $\beta_{1}=0.2$

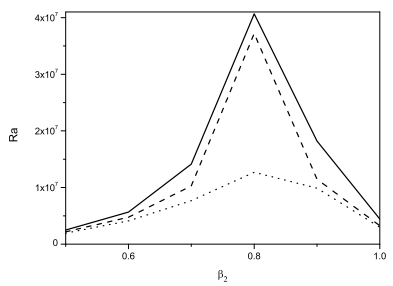

(d) $\beta_{1}=0.4$

Figure 2. Visual representation of linear instability (solid line) and generalized energy method (dashed line) and weighted energy method (doted line)thresholds, with critical Rayleigh number plotted against $\beta_{2}$, for $R_{c}=5$.

Figure 2 gives a visual representation of the linear instability and nonlinear stability (generalized energy method and weighted energy method) boundaries, 
with critical thermal Rayleigh number $R a$ plotted against $\beta_{2}$ for different values of $\beta_{1}$. The remaining parameter are held fixed at $R_{c}=5$.. It is clear that the region of potential subcritical instabilities between the linear instability and nonlinear stability thresholds is considerable and we can conclude that (for the parameter ranges explored) the linear theory not accurately encapsulates the physics of the onset of convection. Thus, the novel instability results are supported by the considerable region of subcritical instabilities.

The nonlinear thresholds clearly display a potentially large region where subcritical instabilities may arise. However, we have computed these in the $\left(\beta_{1}, \beta_{1}\right)$ region where a very rapid rise in the Rayleigh number is observed. $\mathrm{A}$ similar discrepancy in linear versus nonlinear thresholds was observed earlier by Straughan [20,23] in a thermal convection model, although the situation there was not as complicated as the present. For $\beta_{1}=0.1,0.2$, it is clear that $R a$ increases with increasing $\beta_{2}$ (for $\beta_{2}<0.7$ ) which refers to the stabilizing effect of increasing $\beta_{1}$ and $\beta_{2}$. However, when $\beta_{2}>0.7$, we notice that $R a$ decreases with increasing $\beta_{1}$ which refers to the destabilize effect of increasing $\beta_{1}$ and $\beta_{2}$. For $\beta_{1}=0.3,0.4$ and when $\beta_{2}<0.8$, Figure 2 shows that $R a$ increases with increasing $\beta_{2}$, while, when $\beta_{2}>0.8, R a$ decreases with increasing $\beta_{1}$.

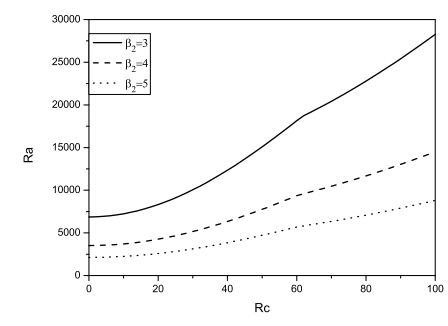

(a) $\beta_{1}=1$

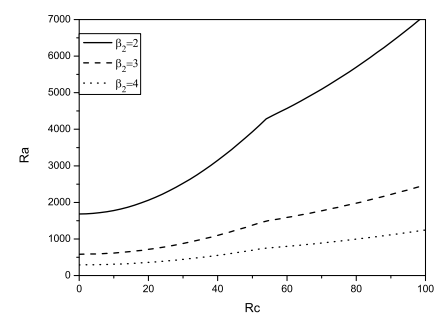

(b) $\beta_{1}=2$

Figure 3. Visual representation of linear instability thresholds, with critical Rayleigh number plotted against the solute Rayleigh number $R_{c}$.

The thresholds of the numerical linear instability results are presented in Figure 3. In this figure, the kinks in the graphs represent the points at which convection switches from steady convection $(\sigma=0)$ to oscillatory convection $\left(\sigma_{r}=0, \sigma_{i} \neq 0\right)$. The onset of both types of convection appear to follow a linear relationship between $R_{c}$ and $R a_{L}$. The results show the effect of increasing $\beta_{2}$ on the critical Rayleigh number for various values of $\beta_{1}$. Figure 3 demonstrates that $R a$ increases with increasing $\beta_{1}$ and $\beta_{2}$ which shows the stabilizing effect of these coefficients. It is interesting to note that in Figure 3 and for $\beta_{1}=1$, the stationary convection become dominator in the linear instability thresholds when $R_{c}<60$ and when $R_{c} \geq 60$, the oscillatory modes become present in the linear instability thresholds. However, for $\beta_{1}=2$ the oscillatory mode become present in the linear instability thresholds when $R_{c} \geq 55$, for $\beta_{2}=2$ and $R_{c} \geq 53$, for $\beta_{2}=3,4$, otherwise, the stationary mode appears in the linear instability. 


\section{Conclusions}

In this paper we have explored the problem of double-diffusive convection in a horizontal plane fluid layer when there is a heat sink/source which is linear in the vertical coordinate which is in the opposite direction to gravity, utilising linear stability analysis and non-linear stability by means of energy functional. A comparison between the linear stability thresholds and energy stability thresholds is made. In both cases the thermal Rayleigh number is evaluated for different combinations of the flow governing parameters. The results indicate that the increasing in the values of $\beta_{1}$ and $\beta_{2}$ has a stabilizing effect in the linear and nonlinear cases up to a certain limit. However, when the values of $\beta_{1}$ and $\beta_{2}$ beyond this limit, this causes a strong destabilization effect on the results.

\section{Acknowledgements}

This work was supported by the Iraqi ministry of higher education and scientific research. The author would like to thank two anonymous referees for their pointed remarks that have led to improvements in the manuscript.

\section{References}

[1] M. Bulícek, J. Málek and K.R. Rajagopal. Naviers slip and evolutionary NavierStokes-like systems with pressure and shear-rate dependent viscosity. Indiana University mathematics journal, 56(1):51-85, 2007.

[2] S. Chandrasekhar. Hydrodynamic and hydromagnetic stability. Courier Corporation, 2013.

[3] Z. Duan. Second-order gaseous slip flow models in long circular and noncircular microchannels and nanochannels. Microfluidics and nanofluidics, 12(5):805-820, 2012. https://doi.org/10.1007/s10404-011-0924-0.

[4] A.J. Harfash. Nonhomogeneous porosity and thermal diffusivity effects on a double-diffusive convection in anisotropic porous media. International Journal of Nonlinear Sciences and Numerical Simulation, 17(5):205-220, 2016. https://doi.org/10.1515/ijnsns-2015-0139.

[5] A.J. Harfash. Resonant penetrative convection in porous media with an internal heat source/sink effect. Applied Mathematics and Computation, 281:323-342, 2016. https://doi.org/10.1016/j.amc.2016.01.006.

[6] A.J. Harfash. Stability analysis for penetrative convection in a fluid layer with throughflow. International Journal of Modern Physics C, 27(09):1650101, 2016. https://doi.org/10.1142/S0129183116501011.

[7] A.J. Harfash. Three dimensional simulations for convection induced by the selective absorption of radiation for the Brinkman model. Meccanica, 51(3):501-515, 2016. https://doi.org/10.1007/s11012-015-0215-z.

[8] A.J. Harfash and A.K. Alshara. On the stationary and oscillatory modes of triply resonant penetrative convection. International Journal of Numerical Methods for Heat \& Fluid Flow, 26(5):1391-1415, 2016. https://doi.org/10.1108/HFF03-2015-0092. 
[9] A.A. Hill and M.R. Morad. Convective stability of carbon sequestration in anisotropic porous media. In Proc. $R$. Soc. A, volume 470, p. 20140373. The Royal Society, 2014. https://doi.org/10.1098/rspa.2014.0373.

[10] A.A. Hill, K.R. Rajagopal and L. Vergori. On the stability and uniqueness of the flow of a fluid through a porous medium. Zeitschrift für angewandte Mathematik und Physik, 67(3):1-12, 2016. https://doi.org/10.1007/s00033-016-0645-z.

[11] O.A. Ladyzhenskaya. New equations for the description of the motions of viscous incompressible fluids, and global solvability for their boundary value problems. Trudy Matematicheskogo Instituta Imeni VA Steklova, 102:85-104, 1967.

[12] O.A. Ladyzhenskaya. On some nonlinear problems in the theory of continuous media. Am. Math. Soc. Transl, 2(70):73-89, 1968.

[13] O.A. Ladyzhenskaya and R.A. Silverman. The mathematical theory of viscous incompressible flow. Gordon \& Breach New York, 1969.

[14] A. Matta, P.A.L. Narayana and A.A. Hill. Double-diffusive HadleyPrats flow in a porous medium subject to gravitational variation. International Journal of Thermal Sciences, 102:300-307, 2016. https://doi.org/10.1016/j.ijthermalsci.2015.10.034.

[15] A. Matta, P.A.L. Narayana and A.A. Hill. Nonlinear thermal instability in a horizontal porous layer with an internal heat source and mass flow. Acta Mechanica, 227(6):1743-1751, 2016. https://doi.org/10.1007/s00707-016-15918.

[16] M. Mharzi, M. Daguenet and S. Daoudi. Thermosolutal natural convection in a vertically layered fluid-porous medium heated from the side. Energy conversion and management, 41(10):1065-1090, 2000.

[17] B. Straughan. Explosive instabilities in mechanics. Springer Science \& Business Media, 1998. https://doi.org/10.1007/978-3-642-58807-5.

[18] B. Straughan. Sharp global nonlinear stability for temperature-dependent viscosity convection. In Proceedings of the Royal Society of London A: Mathematical, Physical and Engineering Sciences, volume 458, pp. 1773-1782. The Royal Society, 2002. https://doi.org/10.1098/rspa.2001.0945.

[19] B. Straughan. The energy method, stability, and nonlinear convection, volume 91. Springer New York, 2004.

[20] B. Straughan. Triply resonant penetrative convection. In Proc. R. Soc. A, p. rspa20120211. The Royal Society, 2012. https://doi.org/10.1098/rspa.2012.0211.

[21] B. Straughan. Porous convection with local thermal non-equilibrium temperatures and with Cattaneo effects in the solid. In Proc. R. Soc. A, volume 469, p. 20130187. The Royal Society, 2013. https://doi.org/10.1098/rspa.2013.0187.

[22] B. Straughan. Anisotropic inertia effect in microfluidic porous thermosolutal convection. Microfluidics and nanofluidics, 16(1-2):361-368, 2014. https://doi.org/10.1007/s10404-013-1208-7.

[23] B. Straughan. Resonant penetrative convection with an internal heat source/sink. Acta Applicandae Mathematicae, 132(1):561-581, 2014. https://doi.org/10.1007/s10440-014-9930-z. 